Commun. Korean Math. Soc. 31 (2016), No. 1, pp. 27-40

http://dx.doi.org/10.4134/CKMS.2016.31.1.027

\title{
CODES BASED ON RESIDUATED LATTICES
}

\author{
Tsafack Surdive Atamewoue, Young Bae Jun, Celestin Lele, \\ Selestin Ndjeya, And SeOK-Zun Song
}

\begin{abstract}
We define the notion of a residuated lattice valued function on a set as Jun and Song have done in $B C K$-algebras. We also investigate related properties of residuated lattice valued function. We establish the codes generated by residuated lattice valued function and conversely we give residuated lattice valued function and residuated lattice obtained by the giving binary block-code.
\end{abstract}

\section{Introduction}

Residuation is a fundamental concept of ordered structures. In this note, we consider the consequences of adding a residuated monoid operation to lattice. The residuated lattices have been studied in several brances of mathematics. In computer science, a block code is a type of channel coding. It adds redundancy to a message so that, at the receiver, one can decode with minimal errors, provided that the information rate would not exceed the channel capacity. The main characterization of a block code is that it is a fixed length channel code. Typically, a block code takes $k$-digit information word, and transforms this into $n$-digit codeword. Block coding is the primary type of channel coding used in earlier mobile communication systems. A block code is a code which encodes strings formed an alphabet set $\mathbb{A}$ into code words by encoding each letter of $\mathbb{A}$ separately. One of the recent applications of $B C K$-algebras was given in the coding theory. In coding theory, a block code is an error-correcting code which encode data in blocks. In 2011, Jun and Song [3] studied codes based on $B C K$ algebras. They constructed a finite binary block-code associated to a finite $B C K$-algebra, and at the end of the paper they put the question if the converse of this statement is also true. Flaut [2] prove that, in some circumstances, the converse of the above statement is also true. Borumand Saeid et al. [4] presented new connections between $B C K$-algebras and binary block codes.

In this paper, we first introduce the notion of residuated lattice valued functions and investigate several properties. We establish block-codes by using

Received April 7, 2015; Revised July 23, 2015.

2010 Mathematics Subject Classification. 03G25, 94B60.

Key words and phrases. RL-function, cut function, cut subset. 
the notion of residuated lattice valued functions. We show that every finite residuated lattice determines a block-code.

\section{Preliminaries}

A residuated lattice, (in short $\mathrm{RL}$ ) is an algebra $(A, \wedge, \vee, \odot, \rightarrow, 0,1)$ of type $(2,2,2,2,0,0)$, equipped with an order $\leq$ satisfying the following conditions:

(I) $(A, \wedge, \vee, 0,1)$ is a bounded lattice,

(II) $(A, \odot, 1)$ is a commutative ordered monoid,

(III) $\odot$ and $\rightarrow$ form an adjoint pair, i.e., $c \leq a \rightarrow b$ if and only if $a \odot c \leq b$ for all $a, b, c \in A$.

The relation between the pair of operations $\odot$ and $\rightarrow$ expressed by (III), is a particular case of the law of residuation, or Galois correspondence (see [5]) and for every $x, y \in A, x \rightarrow y=\sup \{z \in A: x \odot z \leq y\}$.

Any residuated lattices $A$ satisfies the following axioms for all $x, y, z \in A$

$\left(a_{1}\right) 1 \rightarrow x=x, x \rightarrow x=1, x \rightarrow 1=1$,

$\left(a_{2}\right) x \leq y$ if and only if $x \rightarrow y=1$,

$\left(a_{3}\right) x \rightarrow y \leq(z \rightarrow x) \rightarrow(z \rightarrow y)$,

$\left(a_{4}\right) x \rightarrow y \leq(y \rightarrow z) \rightarrow(x \rightarrow z)$,

$\left(a_{5}\right) x \leq y \Rightarrow z \rightarrow x \leq z \rightarrow y, y \rightarrow z \leq x \rightarrow z$.

\section{RL-valued functions}

In what follows, let $X$ and $A$ denote a nonempty set and a residuated lattice, respectively, unless otherwise specified.

Definition 3.1. A mapping $\tilde{f}: X \rightarrow A$ is called a residuated lattice valued function (briefly, RL-function) on $X$.

Definition 3.2. A cut function of $\tilde{f}$, for $a \in A$, is defined to be a mapping $\widetilde{f}_{a}: X \rightarrow\{0,1\}$, such that $(\forall x \in X)\left(\widetilde{f}_{a}(x)=1 \Leftrightarrow a \rightarrow \widetilde{f}(x)=1\right)$.

Remark 3.3. (i) $\tilde{f}_{a}$ is the characteristic function of the following subset of $X$, called a cut subset or an $a$-cut of $\widetilde{f}: X_{a}:=\{x \in X \mid a \rightarrow \widetilde{f}(x)=1\}$.

(ii) $X_{0}=X$ and $X_{1}=\{x \in X \mid \tilde{f}(x)=1\}$.

Example 3.4. Let $X=\{x, y, z\}$ and $A=\{0, a, b, c, 1\}$ with $0<a, b<c<$ 1 , but $a, b$ are incomparable. Then $A$ is a residuated lattice relative to the following operations:

\begin{tabular}{c|ccccc}
$\rightarrow$ & 0 & $a$ & $b$ & $c$ & 1 \\
\hline 0 & 1 & 1 & 1 & 1 & 1 \\
$a$ & $b$ & 1 & $b$ & 1 & 1 \\
$b$ & $a$ & $a$ & 1 & 1 & 1 \\
$c$ & 0 & $a$ & $b$ & 1 & 1 \\
1 & 0 & $a$ & $b$ & $c$ & 1
\end{tabular}

\begin{tabular}{c|ccccc}
$\odot$ & 0 & $a$ & $b$ & $c$ & 1 \\
\hline 0 & 0 & 0 & 0 & 0 & 0 \\
$a$ & 0 & $a$ & 0 & $a$ & $a$ \\
$b$ & 0 & 0 & $b$ & $b$ & $b$ \\
$c$ & 0 & $a$ & $b$ & $c$ & $c$ \\
1 & 0 & $a$ & $b$ & $c$ & 1
\end{tabular}


Consider an RL-function $\tilde{f}$ on $X$ as follows:

$$
\tilde{f}: X \rightarrow A, u \mapsto \begin{cases}a & \text { if } u=x, \\ b & \text { if } u=y, \\ c & \text { if } u=z .\end{cases}
$$

Then its cut subsets are

$$
X_{0}=X, X_{a}=\{x, z\}, X_{b}=\{y, z\}, X_{c}=\{z\}, X_{1}=\emptyset .
$$

Proposition 3.1. Every $R L$-function $\tilde{f}: X \rightarrow A$ on $X$ is represented by the supremum of the set $\left\{a \in A \mid \widetilde{f}_{a}(x)=1\right\}$, that is,

$$
(\forall x \in X)\left(\widetilde{f}(x)=\sup \left\{a \in A \mid \widetilde{f}_{a}(x)=1\right\}\right) .
$$

Proof. For any $x \in X$, let $\widetilde{f}(x)=r \in A$. Then $r \rightarrow \widetilde{f}(x)=r \rightarrow r=1$, and so $\widetilde{f}_{r}(x)=1$, i.e., $r \in\left\{a \in A \mid \widetilde{f}_{a}(x)=1\right\}$. Let $a \in A$ be such that $\widetilde{f}_{a}(x)=1$. Then $1=a \rightarrow \widetilde{f}(x)=a \rightarrow r$, and thus $a \leq r$. Hence $r=\sup \left\{a \in A \mid \widetilde{f}_{a}(x)=\right.$ $1\}$. Therefore $\left.\widetilde{f}(x)=\sup \left\{a \in A \mid \widetilde{f}_{a}(x)=1\right\}\right)$.

Proposition 3.2. If $\tilde{f}: X \rightarrow A$ is an RL-function on $X$, then

$$
(\forall x \in X)\left(\widetilde{f}(x)=\sup \left\{a \mapsto \widetilde{f}_{a}(x) \mid a \in A\right\}\right),
$$

where $a \mapsto \widetilde{f}_{a}(x)=\left\{\begin{array}{cc}a & \widetilde{f}_{a}(x)=1, \\ 0 & \text { otherwise. }\end{array}\right.$

Proof. For any $x \in X$, let $\widetilde{f}(x)=r$. Then $r \rightarrow \widetilde{f}(x)=1$, and thus $\widetilde{f}_{r}(x)=1$. Hence $r \mapsto \widetilde{f}_{r}(x) \in\left\{a \mapsto \widetilde{f}_{a}(x) \mid a \in A\right\}$. Let $a \mapsto \widetilde{f}_{a}(x) \in A$ be such that $a \in A$. Then

$$
\left(a \mapsto \widetilde{f}_{a}(x)\right) \rightarrow r= \begin{cases}a \rightarrow r=a \rightarrow \widetilde{f}(x)=1, & \text { if } \widetilde{f}_{a}(x)=1 ; \\ 0 \rightarrow r=1, & \text { if } \widetilde{f}_{a}(x)=0 .\end{cases}
$$

Thus $\left(a \mapsto \widetilde{f}_{a}(x)\right) \rightarrow r=1$, and so $\left(a \mapsto \widetilde{f}_{a}(x)\right) \leq r$. Since

$$
r=r \mapsto \widetilde{f}_{r}(x) \in\left\{a \mapsto \widetilde{f}_{a}(x) \mid a \in A\right\},
$$

it follows that $\widetilde{f}(x)=r=\sup \left\{a \mapsto \widetilde{f}_{a}(x) \mid a \in A\right\}$.

Proposition 3.3. Let $\tilde{f}: X \rightarrow A$ be an RL-function on $X$. Then

$$
(\forall a, b \in A)\left(a \rightarrow b=1 \Rightarrow X_{b} \subseteq X_{a}\right) .
$$

Proof. Let $a, b \in A$ be such that $a \rightarrow b=1$. Let $x \in X_{b}$. Then $b \rightarrow \widetilde{f}(x)=1$, and so $b \leq \widetilde{f}(x)$. It follows from $\left(a_{5}\right)$ that $a \rightarrow b \leq a \rightarrow \widetilde{f}(x)$, and so that

$$
a \rightarrow \tilde{f}(x)=1 \rightarrow(a \rightarrow \tilde{f}(x))=(a \rightarrow b) \rightarrow(a \rightarrow \widetilde{f}(x))=1 .
$$

Therefore $x \in X_{a}$, which shows that $X_{b} \subseteq X_{a}$.

Proposition 3.4. Let $\tilde{f}: X \rightarrow A$ be an RL-function on $X$. Then 
(i) $(\forall x, y \in X)\left(\widetilde{f}(x) \neq \widetilde{f}(y) \Leftrightarrow X_{\widetilde{f}(x)} \neq X_{\widetilde{f}(y)}\right)$.

(ii) $(\forall a \in A)(\forall x \in X)\left(a \rightarrow \widetilde{f}(x)=1 \Leftrightarrow X_{\widetilde{f}(x)} \subseteq X_{a}\right)$.

Proof. (i) If $\tilde{f}(x) \neq \tilde{f}(y)$ for all $x, y \in X$, then $\tilde{f}(x) \rightarrow \widetilde{f}(y) \neq 1$ or $\tilde{f}(y) \rightarrow$ $\widetilde{f}(x) \neq 1$. Hence

$$
X_{\widetilde{f}(x)}=\{z \in X \mid \widetilde{f}(x) \rightarrow \widetilde{f}(z)=1\} \neq\{z \in X \mid \widetilde{f}(y) \rightarrow \widetilde{f}(z)=1\}=X_{\widetilde{f}(y)} .
$$

Conversely, let $x, y \in X$ be such that $X_{\widetilde{f}(x)} \neq X_{\widetilde{f}(y)}$. Assume that $\widetilde{f}(x)=$ $\widetilde{f}(y)$. If $z \in X_{\widetilde{f}(x)}$, then

$$
1=\tilde{f}(x) \rightarrow \widetilde{f}(z)=\tilde{f}(y) \rightarrow \widetilde{f}(z)
$$

and so $z \in X_{\widetilde{f}(y)}$. Thus $X_{\widetilde{f}(x)} \subseteq X_{\widetilde{f}(y)}$. Similarly, $X_{\widetilde{f}(y)} \subseteq X_{\widetilde{f}(x)}$. This is a contradiction. Therefore $\tilde{f}(x) \neq \tilde{f}(y)$.

(ii) Let $a \in A$ and $x \in X$ be such that $a \rightarrow \widetilde{f}(x)=1$. Then $X_{\widetilde{f}(x)} \subseteq X_{a}$ by Proposition 3.3.

Conversely, let $a \in A$ and $x \in X$ be such that $X_{\widetilde{f}(x)} \subseteq X_{a}$. If $a \rightarrow \widetilde{f}(x) \neq 1$, then $x \notin X_{a}$. The identity $\widetilde{f}(x) \rightarrow \widetilde{f}(x)=1$ implies $x \in X_{\widetilde{f}(x)}$. Hence $X_{\widetilde{f}(x)}$ is not include in $X_{a}$, which is a contradiction. Therefore $a \rightarrow \widetilde{f}(x)=1$.

Corollary 3.5. Let $\tilde{f}: X \rightarrow A$ be an RL-function on $X$. Then

$$
(\forall x, y \in A)\left(\widetilde{f}(x) \rightarrow \widetilde{f}(y)=1 \Leftrightarrow X_{\widetilde{f}(y)} \subseteq X_{\widetilde{f}(x)}\right) .
$$

Proof. It is straightforward by Proposition 3.4(ii).

For an RL-function $\tilde{f}: X \rightarrow A$ on $X$, consider the following sets:

$$
X_{A}:=\left\{X_{a} \mid a \in A\right\} \text { and } \widetilde{f}_{A}:=\left\{\tilde{f}_{a} \mid a \in A\right\} .
$$

Proposition 3.6. Let $\tilde{f}: X \rightarrow A$ be an $R L$-function on $X$. Then

$$
(\forall B \subseteq A)\left(X_{\text {sup }\{b \mid b \in B\}}=\bigcap\left\{X_{b} \mid b \in B\right\}\right) .
$$

Proof. Note that there exists the supermum of $B$ in $A$ for any $B \subseteq A$. We have

$$
\begin{aligned}
x \in X_{\sup \{b \mid b \in B\}} & \Rightarrow \sup \{b \mid b \in B\} \rightarrow \tilde{f}(x)=1 \\
& \Rightarrow(\forall b \in B)(b \rightarrow \tilde{f}(x)=1) \\
& \Rightarrow(\forall b \in B)\left(x \in X_{b}\right) \\
& \Rightarrow x \in \bigcap\left\{X_{b} \mid b \in B\right\} .
\end{aligned}
$$

Thus $X_{\text {sup }\{b \mid b \in B\}} \subseteq \bigcap\left\{X_{b} \mid b \in B\right\}$. Also, we have

$$
\begin{aligned}
x \in \bigcap\left\{X_{b} \mid b \in B\right\} & \Rightarrow(\forall b \in B)\left(x \in X_{b}\right) \\
& \Rightarrow(\forall b \in B)(b \rightarrow \widetilde{f}(x)=1)
\end{aligned}
$$




$$
\begin{aligned}
& \Rightarrow(\forall b \in B)(b \leq \tilde{f}(x)) \\
& \Rightarrow \sup \{b \mid b \in B\} \leq \widetilde{f}(x) \\
& \Rightarrow \sup \{b \mid b \in B\} \rightarrow \widetilde{f}(x)=1 \\
& \Rightarrow x \in X_{\sup \{b \mid b \in B\}} .
\end{aligned}
$$

Thus $\bigcap\left\{X_{b} \mid b \in B\right\} \subseteq X_{\sup \{b \mid b \in B\}}$. This completes the proof.

Corollary 3.7. Let $\tilde{f}: X \rightarrow A$ be an RL-function on $X$. Then

$$
(\forall a, b \in A)\left(X_{a} \cap X_{b} \in X_{A}\right) .
$$

Proof. Note that for any $a, b \in A$, there exists $\sup \{a, b\}$ in $A$. By Proposition 3.6 ,

$$
X_{\text {sup }\{a, b\}}=\bigcap\left\{X_{t} \mid t \in\{a, b\}\right\}=X_{a} \cap X_{b} .
$$

Since $X_{\sup \{a, b\}} \in X_{A}$, it follows that $X_{a} \cap X_{b} \in X_{A}$.

Proposition 3.8. Let $\tilde{f}: X \rightarrow A$ be an RL-function on $X$. Then

$$
\bigcup\left\{X_{a} \mid a \in A\right\}=X \text {. }
$$

Proof. Note that $\bigcup\left\{X_{a} \mid a \in A\right\}=X_{0} \cup\left\{X_{a} \mid a \in A \backslash\{0\}\right\}$. Since $X_{a} \subseteq X$ for all $a \in A \backslash\{0\}$, it follow that $X_{0} \cup\left\{X_{a} \mid a \in A \backslash\{0\}\right\}=X$, that is, $\bigcup\left\{X_{a} \mid a \in A\right\}=X$.

Proposition 3.9. Let $\widetilde{f}: X \rightarrow A$ be an RL-function on $X$. Then

$$
(\forall x \in X)\left(\bigcap\left\{X_{a} \mid x \in X_{a}\right\} \in X_{A}\right) .
$$

Proof. For any $x \in X$, we know that $x \in X_{a}$ implies $a \rightarrow \widetilde{f}(x)=1$. Let

$$
B=\left\{a \in A \mid \tilde{f}_{a}(x)=1\right\} .
$$

Then $B \subseteq A$, and so

$$
\bigcap\left\{X_{a} \mid x \in X_{a}\right\}=\bigcap\left\{X_{a} \mid \widetilde{f}_{a}(x)=1\right\}=X_{\sup \left\{a \in A \mid \widetilde{f}_{a}(x)=1\right\}} \in X_{A}
$$

by Proposition 3.6.

Let $\tilde{f}: X \rightarrow A$ be an RL-function on $X$ and let $\sim$ be a binary relation on $A$ defined by

$$
(\forall a, b \in A)\left(a \sim b \Leftrightarrow X_{a}=X_{b}\right) .
$$

Remark 3.5. The binary relation $\sim$ is an equivalence relation on $A$.

Consider the sets

$$
\widetilde{f}(X):=\{a \in A \mid \widetilde{f}(x)=a \text { for some } x \in X\}
$$

and $[a):=\{x \in A \mid a \rightarrow x=1\}$ for $a \in A$. 
Proposition 3.10. For an RL-function $\tilde{f}: X \rightarrow A$ on $X$, we have

$$
(\forall a, b \in A)(a \sim b \Leftrightarrow[a) \cap \widetilde{f}(X)=[b) \cap \widetilde{f}(X)) .
$$

Proof. For any $a, b \in A$, we have

$$
\begin{aligned}
a \sim b & \Leftrightarrow X_{a}=X_{b} \\
& \Leftrightarrow(\forall x \in X)(a \rightarrow \widetilde{f}(x)=1 \Leftrightarrow b \rightarrow \widetilde{f}(x)=1) \\
& \Leftrightarrow\{x \in X \mid \widetilde{f}(x) \in[a)\}=\{x \in X \mid \widetilde{f}(x) \in[b)\} \\
& \Leftrightarrow[a) \cap \widetilde{f}(X)=[b) \cap \widetilde{f}(X) .
\end{aligned}
$$

This completes the proof.

\section{Codes generated by RL-functions}

For any $x \in A$, let $x / \sim$ denote the equivalence class containing $x$, that is,

$$
x / \sim:=\{y \in A \mid x \sim y\} .
$$

Lemma 4.1. Let $\tilde{f}: X \rightarrow A$ be an $R L$-function on $X$. For every $x \in X$, we have $\widetilde{f}(x)=\sup \{\widetilde{f}(x) / \sim\}$, that is, $\widetilde{f}(x)$ is the greatest element of the $\sim$-class to which it belongs.

Proof. We have

$$
\begin{aligned}
\widetilde{f}(x) & =\sup \left\{a \in A \mid \tilde{f}_{a}(x)=1\right\} \\
& =\sup \{a \in A \mid a \rightarrow \widetilde{f}(x)=1\} \\
& =\sup \{a \in A \mid a \in \widetilde{f}(x) / \sim\} \\
& =\sup \{\tilde{f}(x) / \sim\} .
\end{aligned}
$$

This completes the proof.

Construction of the code: Let $X=\{1,2, \ldots, n\}$ and $A$ be a finite residuated lattice. Every RL-function $\tilde{f}: X \rightarrow A$ on $X$ determines a binary block-code $C$ of length $n$ in the following way:

To every $a / \sim$, where $a \in A$, there corresponds a codeword $c_{a}=a_{1} a_{2} \cdots a_{n}$ such that

$$
a_{i}=j \Leftrightarrow \widetilde{f}_{a}(i)=j
$$

for $i \in A$ and $j \in\{0,1\}$. It is clear that $C$ is a binary block-code of length $n$. Let $c_{a}=a_{1} a_{2} \cdots a_{n}$ and $c_{b}=b_{1} b_{2} \cdots b_{n}$ be two codewords belonging to a binary block-code $C$. Define an order relation $\leq_{c}$ on the set of codewords belonging to a binary block-code $C$ as follows:

$$
c_{a} \leq_{c} c_{b} \Leftrightarrow b_{i} \leq a_{i} \text { for } i=1,2, \ldots, n .
$$


Example 4.1. Let $A$ be the residuate lattice with the universe $\{0, a, b, c, d, 1\}$ such that $0<a, b<c<1$ and $0<b<d<1$, but $a$ and $b$ are incomparable, and $c$ and $d$ are incomparable. The operations of implication and multiplication are given by the tables below:

\begin{tabular}{c|cccccc}
$\rightarrow$ & 0 & $a$ & $b$ & $c$ & $d$ & 1 \\
\hline 0 & 1 & 1 & 1 & 1 & 1 & 1 \\
$a$ & $d$ & 1 & $d$ & 1 & $d$ & 1 \\
$b$ & $c$ & $c$ & 1 & 1 & 1 & 1 \\
$c$ & $b$ & $c$ & $d$ & 1 & $d$ & 1 \\
$d$ & $a$ & $a$ & $c$ & $c$ & 1 & 1 \\
1 & 0 & $a$ & $b$ & $c$ & $d$ & 1
\end{tabular}

\begin{tabular}{c|cccccc}
$\odot$ & 0 & $a$ & $b$ & $c$ & $d$ & 1 \\
\hline 0 & 0 & 0 & 0 & 0 & 0 & 0 \\
$a$ & 0 & $a$ & 0 & $a$ & 0 & $a$ \\
$b$ & 0 & 0 & 0 & 0 & $b$ & $b$ \\
$c$ & 0 & $a$ & 0 & $a$ & $b$ & $c$ \\
$d$ & 0 & 0 & $b$ & $b$ & $d$ & $d$ \\
1 & 0 & $a$ & $b$ & $c$ & $d$ & 1
\end{tabular}

Let $\tilde{f}: A \rightarrow A$ be an RL-function on $A$ given by

$$
\tilde{f}=\left(\begin{array}{llllll}
0 & a & b & c & d & 1 \\
0 & a & b & c & d & 1
\end{array}\right) .
$$

Then

\begin{tabular}{c|cccccc}
$\widetilde{f}_{x}$ & 0 & $a$ & $b$ & $c$ & $d$ & 1 \\
\hline 0 & 1 & 1 & 1 & 1 & 1 & 1 \\
$a$ & 0 & 1 & 0 & 1 & 0 & 1 \\
$b$ & 0 & 0 & 1 & 1 & 1 & 1 \\
$c$ & 0 & 0 & 0 & 1 & 0 & 1 \\
$d$ & 0 & 0 & 0 & 0 & 1 & 1 \\
1 & 0 & 0 & 0 & 0 & 0 & 1
\end{tabular}

Thus $C=\{111111,010101,001111,000101,000011,000001\}$.
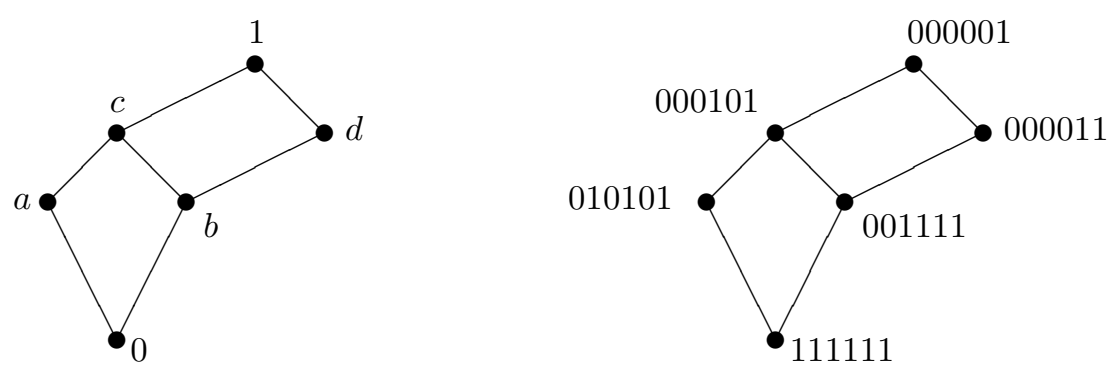

Figure 1. Partial ordering $\leq$, Order relation $\leq_{c}$

Example 4.2. Let $A:=\{0, a, b, c, d, e, f, g, 1\}$ be a set with $0<a<b<e<$ $1,0<c<f<g<1, a<d<g, c<d<e$, but $\{a, c\},\{b, d\},\{d, f\},\{b, f\}$ and $\{e, g\}$, respectively, are incomparable. We define operations $\odot$ and $\rightarrow$ on $A$ by the Cayley tables (see Table 1 and Table 2). Then $A$ is a residuated lattice (see [1]). For a set $X=\left\{x_{1}, x_{2}, x_{3}, x_{4}, x_{5}, x_{6}, x_{7}, x_{8}, x_{9}\right\}$, consider an 
RL-function $\widetilde{f}$ on $X$ as follows:

$$
\widetilde{f}: X \rightarrow A, u \mapsto \begin{cases}1 & \text { if } u=x_{1}, \\ g & \text { if } u=x_{2}, \\ f & \text { if } u=x_{3}, \\ e & \text { if } u=x_{4}, \\ d & \text { if } u=x_{5}, \\ c & \text { if } u=x_{6}, \\ b & \text { if } u=x_{7}, \\ a & \text { if } u=x_{8}, \\ 0 & \text { if } u=x_{9} .\end{cases}
$$

TABLE 1. Cayley table for the "๑"-operation

\begin{tabular}{c|lllllllll}
\hline$\odot$ & 0 & $a$ & $b$ & $c$ & $d$ & $e$ & $f$ & $g$ & 1 \\
\hline 0 & 0 & 0 & 0 & 0 & 0 & 0 & 0 & 0 & 0 \\
$a$ & 0 & 0 & $a$ & 0 & 0 & $a$ & 0 & 0 & $a$ \\
$b$ & 0 & $a$ & $b$ & 0 & $a$ & $b$ & 0 & $a$ & $b$ \\
$c$ & 0 & 0 & 0 & 0 & 0 & 0 & $c$ & $c$ & $c$ \\
$d$ & 0 & 0 & $a$ & 0 & 0 & $a$ & $c$ & $c$ & $d$ \\
$e$ & 0 & $a$ & $b$ & 0 & $a$ & $b$ & $c$ & $d$ & $e$ \\
$f$ & 0 & 0 & 0 & $c$ & $c$ & $c$ & $f$ & $f$ & $f$ \\
$g$ & 0 & 0 & $a$ & $c$ & $c$ & $d$ & $f$ & $f$ & $g$ \\
1 & 0 & $a$ & $b$ & $c$ & $d$ & $e$ & $f$ & $g$ & 1 \\
\hline
\end{tabular}

TABLE 2. Cayley table for the " $\rightarrow$ "-operation

\begin{tabular}{c|lllllllll}
\hline$\rightarrow$ & 0 & $a$ & $b$ & $c$ & $d$ & $e$ & $f$ & $g$ & 1 \\
\hline 0 & 1 & 1 & 1 & 1 & 1 & 1 & 1 & 1 & 1 \\
$a$ & $g$ & 1 & 1 & $g$ & 1 & 1 & $g$ & 1 & 1 \\
$b$ & $f$ & $g$ & 1 & $f$ & $g$ & 1 & $f$ & $g$ & 1 \\
$c$ & $e$ & $e$ & $e$ & 1 & 1 & 1 & 1 & 1 & 1 \\
$d$ & $d$ & $e$ & $e$ & $g$ & 1 & 1 & $g$ & 1 & 1 \\
$e$ & $c$ & $d$ & $e$ & $f$ & $g$ & 1 & $f$ & $g$ & 1 \\
$f$ & $b$ & $b$ & $b$ & $e$ & $e$ & $e$ & 1 & 1 & 1 \\
$g$ & $a$ & $b$ & $b$ & $d$ & $e$ & $e$ & $g$ & 1 & 1 \\
1 & 0 & $a$ & $b$ & $c$ & $d$ & $e$ & $f$ & $g$ & 1 \\
\hline
\end{tabular}


Then

Hence

\begin{tabular}{c|ccccccccc} 
& $x_{1}$ & $x_{2}$ & $x_{3}$ & $x_{4}$ & $x_{5}$ & $x_{6}$ & $x_{7}$ & $x_{8}$ & $x_{9}$ \\
& 1 & $g$ & $f$ & $e$ & $d$ & $c$ & $b$ & $a$ & 0 \\
\hline$\widetilde{f}_{0}$ & 1 & 1 & 1 & 1 & 1 & 1 & 1 & 1 & 1 \\
$\widetilde{f_{a}}$ & 1 & 1 & 0 & 1 & 1 & 0 & 1 & 1 & 0 \\
$\widetilde{f_{b}}$ & 1 & 0 & 0 & 1 & 0 & 0 & 1 & 0 & 0 \\
$\widetilde{f_{c}}$ & 1 & 1 & 1 & 1 & 1 & 1 & 0 & 0 & 0 \\
$\widetilde{f_{d}}$ & 1 & 1 & 0 & 1 & 1 & 0 & 0 & 0 & 0 \\
$\widetilde{f_{e}}$ & 1 & 0 & 0 & 1 & 0 & 0 & 0 & 0 & 0 \\
$\widetilde{f}_{f}$ & 1 & 1 & 1 & 0 & 0 & 0 & 0 & 0 & 0 \\
$\widetilde{f}_{g}$ & 1 & 1 & 0 & 0 & 0 & 0 & 0 & 0 & 0 \\
$\widetilde{f}_{1}$ & 1 & 0 & 0 & 0 & 0 & 0 & 0 & 0 & 0
\end{tabular}

$$
\begin{aligned}
& C=\{111111111,110110110,100100100,111111000,110110000, \\
& 100100000,111000000,110000000,100000000\}
\end{aligned}
$$

and

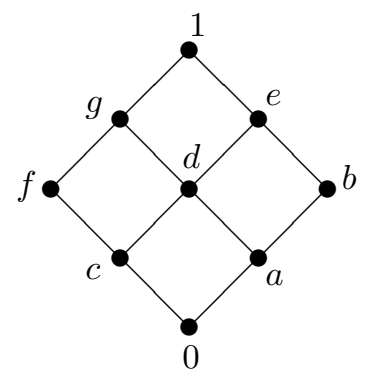

$(S, \leq)$

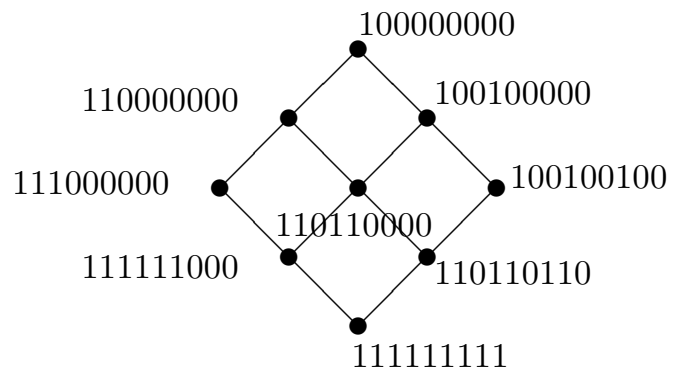

$\left(V, \leq_{c}\right)$

Figure 2. Partial ordering $\leq$, Order relation $\leq_{c}$

Generally, we have the following theorem.

Theorem 4.2. Every finite residuated lattice $A$ determines a block-code $C$ such that $(A, \leq)$ is isomorphic to $\left(C, \leq_{c}\right)$.

Proof. Let $A=\left\{a_{1}, a_{2}, \ldots, a_{n}\right\}$ be a finite residuated lattice in which $a_{1}=0$ and $a_{n}=1$ and let $\tilde{f}: A \rightarrow A$ be the identity RL-function on $A$. The decomposition of $\tilde{f}$ provides a family $\left\{\tilde{f}_{a} \mid a \in A\right\}$ which is the desired code under the order $\leq_{c}$. Let $g: A \rightarrow\left\{\widetilde{f}_{a} \mid a \in A\right\}$ be a function defined by $g(a)=\widetilde{f}_{a}$ for all $a \in A$. By Lemma 4.1 , every $\sim$-class contains exactly one element. Hence $g$ is one-to-one. Let $a, b \in A$ be such that $a \rightarrow b=1$, i.e., $a \leq b$. Then $X_{b} \subseteq X_{a}$ by Proposition 3.3, which means that $\widetilde{f}_{a} \leq \widetilde{f}_{b}$. Therefore $g$ is an isomorphism. 
Definition 4.3. Let $(A, \leq, 0,1)$ be a finite partially ordered set, which is bounded.

We define the following binary relation " $\rightarrow$ " on $A$ as follows:

$$
x=1 \rightarrow x \text { and } x \rightarrow(y \rightarrow z)=y \rightarrow(x \rightarrow z)
$$

for all $x, y, z \in A$.

We define the operation $\odot$, such that $(\odot, \rightarrow)$ forms an adjoint pair, i.e., $z \leq x \rightarrow y$ if and only if $x \odot z \leq y$.

Proposition 4.3 ([5]). With the above operations on $A$, the lattice $(A, \wedge, \vee$, $\odot, \rightarrow, 0,1)$ is a residuated lattice.

Let $C$ be a binary block-code with $n$ codewords of length $n$. We consider the matrix $M_{C}=\left(m_{i j}\right)_{i, j \in\{1,2, \ldots, n\}} \in \mathcal{M}_{n}(\{0,1\})$ with the rows consisting of the codeword of $C$. This matrix is called the matrix associated to the code $C$.

Proposition 4.4. With the above notations, if the codeword $\underbrace{11 \cdots 1}_{n \text {-times }}$ is in $C$ and the matrix $M_{C}$ is upper triangular with $m_{i i}=1$ for all $i \in\{1,2, \ldots, n\}$, there are a set $X$ with $n$ elements, a residuated lattice $A$ and an $R L$-function $\tilde{f}: X \rightarrow A$ on $X$ such that $\tilde{f}$ determines $C$.

Proof. We consider the lexicographic order, denote by $\leq_{l e x}$, on $C$. It is clear that $\left(C, \leq_{l e x}\right)$ is a totally ordered set. Let $C=\left\{w_{1}, w_{2}, \ldots, w_{n}\right\}$, with $w_{1} \leq_{l e x}$ $w_{2} \leq_{\text {lex }} \cdots \leq_{\text {lex }} w_{n}$. This implies that $w_{1}=\underbrace{11 \cdots 1}_{n \text {-times }}$ and $w_{n}=\underbrace{00 \cdots 0}_{(n-1) \text {-times }} 1$.

On $C$, we define a partial order $\leq_{c}$ as in construction of the code by the RL-function. Now, $\left(C, \leq_{c}\right)$ is a partially ordered set with $w_{1} \leq_{c} w_{i} \leq_{c} w_{n}$, $i \in\{1,2, \ldots, n\}$. We remark that $w_{1}$ correspond to 0 and $w_{n}$ correspond to 1 in $A$. Hence $\left(C ; \leq_{c}, 0,1\right)$ is a bounded lattice. We define on $\left(C ; \leq_{c}, 0,1\right)$ a binary relation $\rightarrow$ and the operation $\odot$ as Definition 4.3. Then $A=\left(C ; \leq_{c}, 0,1, \rightarrow, \odot\right)$ is a residuated lattice and $C$ is isomorphic to $A$. We consider $X=C$ and the identity map $\widetilde{f}: X \rightarrow A, w \mapsto w$, as an RL-function on $X$. The decomposition of $\tilde{f}$ provides a family

$$
C_{A}=\left\{\tilde{f}_{r}: X \rightarrow\{0,1\} \mid \tilde{f}_{r}(x)=1 \Leftrightarrow r \rightarrow \widetilde{f}(x)=1, \forall x \in X, r \in A\right\} .
$$

This family is the binary block-code $C$ relative to the order relation $\leq_{c}$.

Proposition 4.5. Let $A=\left(a_{i, j}\right)_{i \in\{1,2, \ldots, n\}, j \in\{1,2, \ldots, m\}} \in \mathcal{M}_{n, m}(\{0,1\})$ be a matrix with rows lexicographic ordered in the descending sense. Starting from this matrix, we can find a matrix $B=\left(b_{i, j}\right)_{i, j \in\{1,2, \ldots, q\}} \in \mathcal{M}_{q}(\{0,1\}), q=$ $n+m$, such that $B$ is an upper triangular matrix, with $b_{i i}=1, \forall i \in\{1,2, \ldots, q\}$ and $A$ becomes a submatrix of the matrix $B$.

Proof. We insert in the left side of the matrix $A$ (from the right to the left) the following $n$ new columns of the form

$$
\underbrace{00 \cdots 01}_{n}, \underbrace{00 \cdots 10}_{n}, \ldots, \underbrace{10 \cdots 00}_{n} .
$$


It results a new matrix $L$ with $n$ rows and $n+m$ columns. Now, we insert the bottom of the matrix $L$ the following $m$ rows:

$$
\underbrace{00 \cdots 0}_{n} \underbrace{11 \cdots 11}_{m}, \underbrace{00 \cdots 0}_{n+1} \underbrace{11 \cdots 1}_{m-1}, \cdots, \underbrace{00 \cdots 0}_{n+m-1} 1 .
$$

We obtained the desired matrix $B$.

Proposition 4.6. With the above notations, we consider $C$ a binary blockcode with $n$ codewords of length $m, n \neq m$, or a block-code with $n$ codewords of length $n$ such that the codeword $\underbrace{11 \cdots 1}_{n \text {-times }}$ is not in $C$, or a block-code with $n$ codewords of length $n$ such that the matrix $M_{C}$ is not upper triangular. There are a natural number $q \geq \max \{m, n\}$, a set $X$ with $m$ elements and an $R L$ function $\tilde{f}: X \rightarrow C_{q}$, where $C_{q}$ denote the residuated lattice with $q$ elements, such that the obtained block-code $C_{C_{n}}$ contains the block-code $C$ as a subset.

Proof. Let $C$ be a binary block-code, $C=\left\{w_{1}, w_{2}, \ldots, w_{n}\right\}$, with codewords of length $m$. We consider the codewords $w_{1}, w_{2}, \ldots, w_{n}$ lexicographic ordered, $w_{1} \leq_{\text {lex }} w_{2} \leq_{\text {lex }} \cdots \leq_{\text {lex }} w_{n}$. Let $M \in \mathcal{M}_{n, m}(\{0,1\})$ be the associated matrix with the rows $w_{1}, w_{2}, \ldots, w_{n}$ in this order. Using Proposition 4.5 , we can extend the matrix $M$ to a square matrix $M^{\prime} \in \mathcal{M}_{p}(\{0,1\}), p=m+n$; such that $M^{\prime}=\left(m_{i j}^{\prime}\right)_{i, j \in\{1,2, \ldots, p\}}$ is an upper triangular matrix with $m_{i i}^{\prime}=1$, for all $i \in\{1,2, \ldots, p\}$. If the first line of the matrix $M^{\prime}$ is not $\underbrace{11 \cdots 1}_{p \text {-times }}$, then we insert the row $\underbrace{11 \cdots 1}_{p+1 \text {-times }}$ as a first row and the $1 \underbrace{00 \cdots 0}_{p \text {-times }}$ as a first column. Let $q=p+1$, applying Proposition 4.4 for the matrix $M^{\prime}$, we obtain a residuated lattice $C_{q}=\left\{x_{1}, x_{2}, \ldots, x_{q}\right\}$, with $x_{1}$ correspond to 0 and $x_{q}$ correspond to 1 , and a binary block-code $C_{C_{q}}$. Assuming that the initial column of the matrix $M$ have in the new matrix $M^{\prime}$ positions $i_{j_{1}}, i_{j_{2}}, \ldots, i_{j_{n}} \in\{1,2, \ldots, q\}$, let $A=\left\{x_{j_{1}}, x_{j_{2}}, \ldots, x_{j_{n}}\right\} \subseteq C_{q}$. The RL-function $\tilde{f}: X \rightarrow C_{q}$ is such that $\tilde{f}\left(x_{j_{i}}\right)=x_{j_{i}}, i \in\{1,2, \ldots, m\}$, determines the binary-block code $C_{q}$ such that $C \subseteq C_{C_{q}}$ as restriction of the RL-function $\tilde{f}: C_{q} \rightarrow C_{q}$ on $X$ such that $\tilde{f}\left(x_{i}\right)=x_{i}$.

Remark 4.4. Propositions 4.4, 4.5 and 4.6 generalized Theorems 3.2, 3.8 and 3.9 in [2] to residuated lattices, with the similar proofs.

The study of residuated lattice have been carried out from both logic and algebraic standpoint. The filter theory plays an important role in studying residuated lattice. From logic point of view various filters have natural interpretation as various set of provable formulas. Filters are called deductive systems by Turunen to emphasize the fact that they correspond to sets of provable formulas and closed with respect to modus ponens. 
The following remark shows that certain block code which can be construct with the residuated lattices is deductive system.

Remark 4.5. Let $V$ be a binary block code with $m$ codewords of length $q$. With the above notations, let $A$ be the associated residuated lattice and $W=$ $\left\{0, w_{1}, \ldots, w_{m+q}\right\}$ the associated binary block code which include the code $V$ (where $\left.1=w_{m+q}\right)$. We consider the code-words $\left(0, w_{1}, w_{2}, \ldots, w_{m+q}\right)$ lexicographic ordered, $0 \geq_{\text {lex }} w_{1} \geq_{\text {lex }} w_{2} \geq_{\text {lex }} \cdots \geq_{\text {lex }} w_{m+q}$. Let $M \in$ $\mathcal{M}_{m+q+1}(\{0,1\})$ be the associated matrix with the rows $\left\{0, w_{1}, w_{2}, \ldots, w_{m+q}\right\}$ in this order. With the above notations, we have that the set

$$
F:=\left\{w_{m+1}, \ldots, w_{m+q}=1\right\}
$$

is a deductive system in the residuated lattice $A$.

Definition 4.6. Let $(S, \leq)$ be a partially ordered set. For $q \in S$, we define a mapping $S_{q}: S \rightarrow\{0,1\}$ such that $(\forall b \in S)\left(S_{q}(b)=1 \Leftrightarrow q \leq b\right)$.

A codeword $v_{x}=x_{1} x_{2} \cdots x_{n}$ of a binary block code $C$ is determine as follow: $x_{i}=j$ if and only if $S_{x}(i)=j$, for $i \in S$ and $j \in\{0,1\}$.

Example 4.7. Let $S=\{0, a, b, c, 1\} ;(0<a, b<c<1)$ (but $a$ and $b$ are incomparable), be a set with a partial order $S$ showed in left figure.

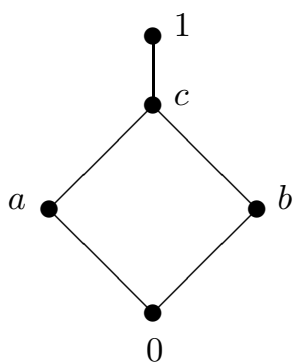

$(S, \leq)$

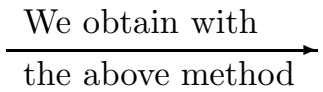
01011

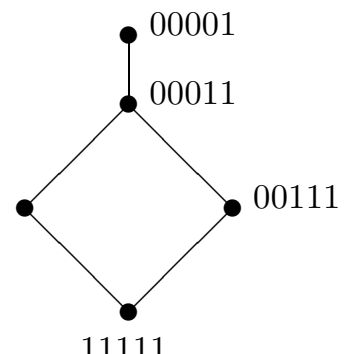

$\left(V, \leq_{c}\right)$

Figure 3. Partial ordering $\leq$, Order relation $\leq_{c}$

\begin{tabular}{c|ccccc}
$S_{x}$ & 0 & $a$ & $b$ & $c$ & 1 \\
\hline$S_{0}$ & 1 & 1 & 1 & 1 & 1 \\
$S_{a}$ & 0 & 1 & 0 & 1 & 1 \\
$S_{b}$ & 0 & 0 & 1 & 1 & 1 \\
$S_{c}$ & 0 & 0 & 0 & 1 & 1 \\
$S_{1}$ & 0 & 0 & 0 & 0 & 1
\end{tabular}

and thus the code obtained by the partially order set $S$ is

$$
V_{S}=\{11111,010111,00111,00011,00001\} .
$$


In the following, we will compute binary block-code based on Jun and Song's method for a residuated lattice. We show that there is a correspondence between the ordered relation on a residuated lattice and a partially ordered set.

Example 4.8. Let $A=\{0, a, b, c, 1\}$ be a residuated lattice with the following Cayley tables, where $0<a, b<c<1$ and $a, b$ are incomparable.

\begin{tabular}{c|ccccc}
$\rightarrow$ & 0 & $a$ & $b$ & $c$ & 1 \\
\hline 0 & 1 & 1 & 1 & 1 & 1 \\
$a$ & $b$ & 1 & $b$ & 1 & 1 \\
$b$ & $a$ & $a$ & 1 & 1 & 1 \\
$c$ & 0 & $a$ & $b$ & 1 & 1 \\
1 & 0 & $a$ & $b$ & $c$ & 1
\end{tabular}

\begin{tabular}{c|ccccc}
$\odot$ & 0 & $a$ & $b$ & $c$ & 1 \\
\hline 0 & 0 & 0 & 0 & 0 & 0 \\
$a$ & 0 & $a$ & 0 & $a$ & $a$ \\
$b$ & 0 & 0 & $b$ & $b$ & $b$ \\
$c$ & 0 & $a$ & $b$ & $c$ & $c$ \\
1 & 0 & $a$ & $b$ & $c$ & 1
\end{tabular}

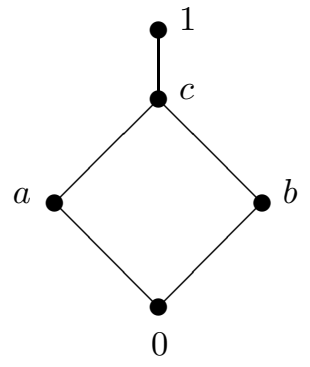

$(S, \leq)$

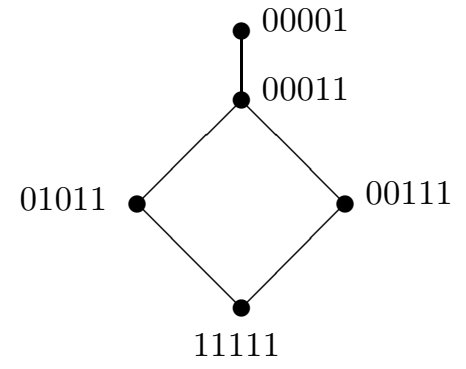

$\left(V, \leq_{c}\right)$

Figure 4. Partial ordering $\leq$, Order relation $\leq_{c}$

Let $\tilde{f}: A \rightarrow A$ be an RL-function on $A$ given by $\tilde{f}=\left(\begin{array}{lllll}0 & a & b & c & 1 \\ 0 & a & b & c & 1\end{array}\right)$. Then

\begin{tabular}{c|ccccc}
$\widetilde{f}_{x}$ & 0 & $a$ & $b$ & $c$ & 1 \\
\hline$\widetilde{f_{0}}$ & 1 & 1 & 1 & 1 & 1 \\
$\widetilde{f}_{a}$ & 0 & 1 & 0 & 1 & 1 \\
$\widetilde{f}_{b}$ & 0 & 0 & 1 & 1 & 1 \\
$\widetilde{f_{c}}$ & 0 & 0 & 0 & 1 & 1 \\
$\widetilde{f}_{1}$ & 0 & 0 & 0 & 0 & 1
\end{tabular}

Thus $C_{A}=\{11111,01011,00111,00011,00001\}$ is a code obtained by the residuated lattice $A$.

Remark 4.9. On a finite lattice which is bounded, we can define a residuated lattice. From the obtained block-codes by the aforesaid methods, it is obvious that $C_{S}=C_{A}$.

Acknowledgements. The authors wish to thank the anonymous reviewers for their valuable suggestions. 


\section{References}

[1] R. Cretan and A. Jeflea, On the lattice of congruence filters of a residuated lattice, An. Univ. Craiova Ser. Mat. Inform. 33 (2006), 174-188.

[2] C. Flaut, BCK-algebras arising from block codes, J. Intell. Fuzzy Systems 28 (2015), no. 4, 1829-1833.

[3] Y. B. Jun and S. Z. Song, Codes based on BCK-algebras, Inform. Sci. 181 (2011), no. 22, 5102-5109.

[4] A. B. Saeid, H. Fatemidokht, C. Flaut, and M. K. Rafsanjani, On codes based on BCKalgebras, Accepted in J. Intell. Fuzzy Syst.

[5] E. Turunen, Mathematics Behind Fuzzy Logic, Physica-Verlag, Heidelberg 1999.

Tsafack Surdive Atamewoue

Departement of MATHEMATiCs

UNIVERSITY OF YAOUNDE 1

Cameroon

E-mail address: surdive@yahoo.fr

Young BAE Jun

Department of Mathematics Education

Gyeongsang National University

JinJu 660-701, KorEA

E-mail address: skywine@gmail.com

Celestin Lele

DePARTEMENT OF MATHEMATiCS

UNIVERSITY OF DSCHANG

Cameroon

E-mail address: celestinlele@yahoo.com

Selestin NdJeya

Departement of Mathematics

Higher Teacher Training College

University OF YAOUNDE 1

CAMERoON

E-mail address: ndjeyas@ens.cm

SEOK-Zun SONG

Department of Mathematics

JEJU NATIONAL UNIVERSity

JEJU 690-756, KorEA

E-mail address: szsong@jejunu.ac.kr 